\title{
Safety of in vitro Diagnostics for Hematology and Coagulation Testing - Analysis of the Reports to the German Competent Authority (BfArM)
}

\author{
Rüdiger Siekmeier Johannes Lütz \\ Bundesinstitut für Arzneimittel und Medizinprodukte (BfArM), Bonn, Germany
}

\section{Key Words}

In vitro diagnostics . Postmarketing surveillance . Coagulation · Hematology

\section{Summary}

The European Directive 98/79/EC on in vitro diagnostic medical devices (IVD) stipulates the marketing and postmarketing surveillance of IVD in the European Economic Area. In case of incidents and field-corrective actions related to IVD, manufacturers have to inform the responsible competent authorities. In Germany, the Federal Institute for Drugs and Medical Devices (BfArM) is the responsible competent authority for most IVD. Only a small subset of IVD for immune hematological and infectiological testing as well as tissue typing specified in Annex II of the Directive is in the responsibility of the Paul-EhrlichInstitute (PEI). Until the end of 2005, the BfArM received a total of 653 notifications regarding IVD. From these, 53 and 34 related to IVD for analysis of coagulation and for hematological testing (without immune hematology), respectively, and were subject of this study. Most of the reports came from manufacturers ( $n=69,79.3 \%$ ), whereas other sources played only minor roles. Product failures of tests and control materials were frequently caused by manufacturing errors and material defects, whereas analyzers were typically affected by software bugs. Based on the investigations of the manufacturers, product failures were confirmed in most cases, and in consequence corrective actions were performed in 71 cases. The latter included frequently customer information, product recalls, modifications in production or quality management, and software upgrades. Our data suggest that the governmental system for postmarketing surveillance is an established and important tool to ensure the safety of IVD.

\section{Schlüsselwörter}

In-vitro-Diagnostika · Marktüberwachung · Gerinnung · Hämatologie

\section{Zusammenfassung}

Die europäische Directive 98/79/EG zu In-vitro-Diagnostika (IVD) regelt Inverkehrbringen und Marktüberwachung von IVD in der Europäischen Union. Bei Vorkommnissen und korrektiven Maßnahmen müssen die Hersteller die zuständigen Überwachungsbehörden informieren. In Deutschland sind dies das Bundesinstitut für Arzneimittel und Medizinprodukte (BfArM) und für einige ausgewählte in Anhang II der Direktive aufgeführte immunhämatologische und infektiologische Produkte das Paul-EhrlichInstitut (PEI). Bis Ende 2005 erhielt das BfArM 653 Meldungen zu IVD. Davon betreffen 53 Produkte zur Gerinnungsuntersuchung und 34 Produkte der Hämatologie (ohne Immunhämatologie). Beide Produktgruppen sind Gegenstand dieser Untersuchung. Die meisten Meldungen ( $\mathrm{n}=69,79,3 \%$ ) kamen von den Herstellern; andere Meldequellen waren von untergeordneter Bedeutung. Kontrollmaterialien und Tests waren typischerweise von Produktions- und Materialfehlern betroffen, während Meldungen zu Analysegeräten häufig Softwarefehler zugrunde lagen. In den meisten Fällen wurden Produktfehler durch die Untersuchungen der Hersteller nachgewiesen; in 71 Fällen erfolgten korrektive Maßnahmen. Diese umfassten meist Kundeninformationen, Rückrufe, Änderungen in Produktion und Qualitätsmanagement sowie Software-Upgrades. Die vorliegenden Daten zeigen, dass es sich bei dem etablierten System zur Marktüberwachung um ein wesentliches, die Sicherheit von IVD gewährleistendes Instrument handelt.

\section{KARGER \\ 우 2007 S. Karger GmbH, Freiburg




\section{Introduction}

The Directive 98/79/EC from 1998 regulates conformity assessment, marketing and postmarketing surveillance of in vitro diagnostics (IVD) in the European Economic Area (EAA) [1]. It has been implemented in Germany by means of the 2nd Amendment on the German Law on Medical Devices at January 1, 2002 (Medizinproduktegesetz; MPG) [2]. The latter has been flanked by the Ordinance on the Medical Devices Vigilance System (Medizinprodukte-Sicherheitsplanverordnung; MPSV) from June 24, 2002 [3]. All these regulations are part of the European medical devices system which ensures safety and quality of medical products including the results of laboratory investigations [4]. In brief, the manufacturers shall be obliged to systematically review the experience gained from devices on the market, to implement corrective actions, where necessary, and to report incidents and recalls to the responsible competent authorities (CAs). According to the MPSV, in Germany also professional operators and users have to report incidents that they observe in using the products to the CA. The same obligation applies to pharmacies and other retail traders if incidents related to over-the-counter (OTC) products sold by them to lay people come to their knowledge [2, 3, 5-7]. In Germany, the Federal Institute for Drugs and Medical Devices (Bundesinstitut für Arzneimittel und Medizinprodukte; BfArM) and the Paul-Ehrlich-Institute (PEI) are responsible for registration and examination of issues related to IVD. The latter is responsible for few IVD for infectiological and immune hematological diagnostics as well as tissue typing as specified in Annex II of Directive 98/79/EC $[1,3,7]$. In detail, these are reagents and reagent products, including related calibrators and control materials, for determining the blood groups (ABO system, rhesus (C, c, D, E, e), anti-Kell, anti-Duffy and anti-Kidd), reagents and reagent products, including related calibrators and control materials, for determining anti-erythrocytic antibodies, reagents and reagent products, including related calibrators and control materials, for determining the HLA tissue groups DR, A and B as well as reagents and reagent products, including related calibrators and control materials, for determining HIV-1 and HIV-2, HTLV-I and HTLV-II, hepatitis B, C and D, cytomegalovirus, Chlamydia, rubella and toxoplasmosis $[1,3,7]$. In evaluating the reports or other relevant information regarding risks, the task of the CA is to characterize the risk (in terms of probability of occurrence of harm and severity of the harm) and to assess it for acceptability. In case of unacceptable risks the necessary corrective action is to be determined. If manufacturers have already taken measures in their own responsibility, the CA has to take a decision on whether or not these are adequate. In Germany, the surveillance of the implementation of corrective actions is in the responsibility of the Federal State authorities BfArM and PEI. In most other European countries other regulations exist [2, 3, 7]. Any necessary field-corrective action performed by the manufacturers must be properly communicated to customers and users. As CE-marked devices in principle enjoy free movement in the entire EEA, there is a need for information to be exchanged between CAs, in particular when corrective action is to be taken. The Directive therefore requires that the European CAs inform each other and the European Commission of cases that lead to corrective actions. Having been informed through a vigilance report, all CAs can then monitor the corrective action in their area of responsibility (if this is deemed to be necessary) and also consider whether similar products of other manufacturers may also be affected by the observed problem [1].

Up to now there are only few published data on the European surveillance system for IVD [8-10]. Because IVD are a very heterogeneous group of tests and analyzers it can be expected that there are large differences between IVD with respect to their use in clinical diagnostics (e.g. clinical chemistry, coagulation testing, hematology, immune hematology, microbiology), the group of users (lay users vs. professional users), the underlying analytical principles (e.g. culture tests, immunological tests, molecular biological tests, hematological tests, coagulation tests, clinical chemistry tests) and/or the structure of the IVD (analyzers vs. tests). For example, there are no data regarding IVD for coagulation testing or hematological analysis which play a significant role in clinical diagnostics and transfusion medicine. Therefore, the aim of this study was to analyze all cases related to IVD specified for coagulation testing and hematological analysis which were reported to the BfArM from January 1999 to December 2005.

\section{Materials and Methods}

Even though the legal base for the competence of BfArM and PEI had been established in 2002 by the implementation of MPG and MPSV [2, 3], both CAs received reports on IVD since 1999 . In this study only cases reported to the BfArM between January 1999 and December 2005 were considered, whereas notifications regarding products in the responsibility of the PEI were not included because these products differ with respect to their conformity assessment procedure prior to marketing [1] and detailed data on reported issues were not available for analysis by the BfArM. Detailed analysis was made only for IVD used for analytics of coagulation (all tests, calibrators and control materials, all analyzers, except for clinical chemistry analyzers with a broad parameter spectrum which allow measurement of very few coagulation parameters only (e.g. d-dimers by immunologic means)) and hematology (all tests, calibrators, control materials and all analyzers). All other IVD, including those for immune hematological testing, tissue typing and robotic sample processors for sample preparation prior to testing, were excluded because they will be subject of other detailed analyses. IVD for hematological analytics were professional-use products only, whereas those for coagulation testing included IVD for professional use as well as OTC products for lay use. Analyses were made separately for the different product groups in order to detect existent differences. Subject of these analyses were the compilation of the report numbers regarding to their sources, the registration of type and number of product failure as well as ascertainment of type and number of the corresponding corrective actions. Statistical analysis was done by means of the chi-square test. 


\begin{tabular}{lcc}
\cline { 2 - 2 } $\begin{array}{l}\text { Table 1. Annual } \\
\text { notifications on }\end{array}$ & Year & Number of reports \\
\cline { 2 - 3 } IVD received by the & 1999 & 13 \\
BfArM (1999-2005, & 2000 & 21 \\
$\mathrm{n}=653)$ & 2001 & 33 \\
& 2002 & 58 \\
& 2003 & 121 \\
& 2004 & 200 \\
& 2005 & 207 \\
\hline
\end{tabular}

\section{Results}

\section{Number of Reports}

Within the observation period the BfArM received an annually increasing number of notifications. After implementation of MPG and MPSV in 2002, a strong increase of reports concerning IVD was observed. A further increase occurred after the end of the transitional period (December 7, 2003) given in the Directive 98/79/EC (table 1). At the end of the observation period the BfArM had received a total number of 653 reports concerning IVD from which $(491,75.2 \%)$ were related to professional-use products and $162(24.8 \%)$ to OTC products (predominantly for measurement of blood glucose [8]).

\section{Product Groups}

53 cases were related to products for coagulation testing. These included 32 reports on tests or control materials (reagents, test kits and sensors $\mathrm{n}=30$; control materials $\mathrm{n}=2$ ) and 10 reports on analyzers and consumables (analyzers $n=8$; consumables (cuvettes, solutions) $\mathrm{n}=2$ ) for professional use as well as 11 cases related to OTC products (table 2). A total of 34 reports was received regarding products for use in hematological diagnostics which were related to tests and control materials (total $n=9$; reagents and test kits $n=6$; control materials $\mathrm{n}=3$ ) as well as analyzers and consumables (total $\mathrm{n}=$ 25; analyzers $n=24$; consumables $n=1$ ) for professional use only (table 3 ).

\section{Sources of Reports}

From all notifications regarding professional-use products for coagulation testing, $35(83.3 \%)$ came from manufacturers and their distributors (only few cases from distributors). A minor number of notifications ( $\mathrm{n}=7,16.7 \%)$ were received from CAs (national authorities $(\mathrm{n}=3)$ and European CAs $(\mathrm{n}=4)$ ). There was obviously no difference with respect to the source of notification between cases related to tests or reagents and those related to analyzers. In case of OTC products for coagulation testing, 7 notifications were received from manufacturers or their distributors, whereas 3 reports came from users (directly from professional users or lay users or via the Drug Commission of the German Pharmaceutical Association who processes the complaints of the pharmacies). One more report was received from a national CA.
Notifications related to products for hematological diagnostics were received from manufacturers and their distributors ( $\mathrm{n}=27,79.4 \%$; only few cases reported from distributors) as well as European or national CAs (total $n=7,20.6 \%$; European $\mathrm{CA} n=6$; national CA $\mathrm{n}=1$ ). There was also no relevant difference with respect to the source of notification between tests or reagents and analyzers.

\section{Frequency and Type of Product Failure}

Investigations by the manufacturers of the professional-use tests and control materials for coagulation testing were able to confirm product failures in 31 (96.9\%) cases (table 2). In 25 of these cases the underlying root cause was identified, but in 6 cases the manufacturers were not able to identify the definite root cause of the product failure even though intensive investigations were performed. Irrespective if the root cause was identified or not, the appearance of the product failures was very similar and typically became obvious by falsely low or falsely high test results, an impaired reagent stability followed by erroneous results or calibration problems also followed by erroneous results. However, the underlying causes of the product failures were very different. Manufacturing errors (e.g. aeration of the vials within the lyophilization process, penetration of humidity into the vial because of incorrectly mounted plugs, fissures of the reagent vials due to an improper process of the machine for closure of the vials, addition of an incorrect amount of zinc to the reagent and suboptimal coating of the macromolecular particles (latex, polystyrene) used in the assay, too short and not yet standardized incubation times affecting the stability of the produced activator complex) and material defects (e.g. unsuitable glass ware, instability or incorrect activity of raw materials (plasma, factor Xa or thrombin)) were most common. Further causes were bacterial or fungal contamination of the reagent (e.g. within the filling process of the vials), labeling errors (incorrect ISI values, incorrect volumes for reagent reconstitution), incorrect instructions for use (erroneous technical data for the sensitivity of the test/analyzer combination, missing information for potential carry-over of reagent-treated samples in the later analytical process), a software bug (misidentification of cartridges due to incorrect data on the chip), a calibration error (provision of incorrect ISI values by the manufacturer) and an analytical interference (lack of linearity in diluted samples).

A total of 10 cases was related to analyzers for coagulation testing $(\mathrm{n}=8)$ or corresponding disposables ( $\mathrm{n}=2$; i.e. cuvettes). In all cases a product failure had been confirmed (table 2 ). Analyzer failures were most frequently caused by software bugs appearing as a risk for sample misidentification, a missing control of the time between pipetting sample or reagent and begin of the incubation which might cause an incorrect incubation time and therefore erroneous results, a non-sufficient time for data acquisition causing an incomplete detection of the reaction, an incorrect algorithm for calculation of the standard curve as well as a missing transfer of a decimal number of the obtained result 
Table 2. Product failures in 53 reported cases on IVD for coagulation testing

\begin{tabular}{|c|c|c|c|c|c|c|}
\hline \multirow[t]{2}{*}{ Product failure } & \multicolumn{2}{|c|}{$\begin{array}{l}\text { Professional-use } \\
\text { tests }^{\mathrm{a}}\end{array}$} & \multicolumn{2}{|c|}{$\begin{array}{l}\text { Professional-use } \\
\text { analyzers }^{\text {b }}\end{array}$} & \multicolumn{2}{|c|}{$\begin{array}{l}\text { OTC tests and } \\
\text { analyzers }\end{array}$} \\
\hline & $\mathrm{n}$ & $\%$ & $\mathrm{n}$ & $\%$ & $\mathrm{n}$ & $\%$ \\
\hline Sum & 32 & 100.0 & 10 & 100.0 & 11 & 100.0 \\
\hline No product failure & 1 & 3.1 & 0 & 0.0 & 2 & 18.2 \\
\hline Unclear product failure & 0 & 0.0 & 0 & 0.0 & 3 & 27.3 \\
\hline User error & 0 & 0.0 & 0 & 0.0 & 0 & 0.0 \\
\hline Product failure & 31 & 96.9 & 10 & 100.0 & 6 & 54.5 \\
\hline Material defect & 6 & & 0 & & 2 & \\
\hline Software bug & 1 & & 6 & & 1 & \\
\hline Calibration error & 1 & & 0 & & 0 & \\
\hline Mechanical error & 0 & & 1 & & 0 & \\
\hline Lack of specification & 0 & & 0 & & 0 & \\
\hline Manufacturing error & 10 & & 1 & & 1 & \\
\hline Incorrect instructions for use & 2 & & 1 & & 0 & \\
\hline Non-microbial contamination & 0 & & 1 & & 0 & \\
\hline Packaging error & 0 & & 0 & & 0 & \\
\hline Microbial contamination & 2 & & 0 & & 0 & \\
\hline Interference by other substances & 1 & & 0 & & 1 & \\
\hline Constructional fault & 0 & & 0 & & 0 & \\
\hline Labeling error & 2 & & 0 & & 1 & \\
\hline Root cause not identified & 6 & & 0 & & 0 & \\
\hline
\end{tabular}

${ }^{a}$ Reagents, calibrators and controls.

${ }^{\mathrm{b}}$ Analyzers and consumables (cuvettes, solutions). from the analyzer to the host software of the lab which was not detected. Two more cases were caused by a mechanical error (failure of the cap piercing system) and a modified sensitivity of the photomultipliers used for the optical reaction detection which was not subject of the instruction for use. Cases related to disposables were caused by a production error in the melting process of the cuvettes and a contamination of the plastic cuvettes with calcium carbonate used in the manufacturing process (table 2).

Cases related to OTC systems for self monitoring of blood coagulation (test strips and analyzers) were subject of 11 reports. Product failures were identified only in $6(54.5 \%)$ cases from which 5 were related to test strips and only 1 to the analyzer (table 2). Cases related to test strips were caused by defects of the used raw materials resulting in a variation of the analytical sensitivity and the results, manufacturing error (desiccation due to a perforated packing), labeling error (declaration of an incorrect conversion factor between the international normalized ratio and the Quick value after a change of the lots), and an analytical interference (interference of lupus anticoagulant). The analyzer-related product failure was caused by an erroneous software algorithm. A product failure was excluded in $2(18.2 \%)$ cases in which the investigations of the manufacturers excluded product defects; in one of these cases the patient's reported complaint stood in contradiction to the potentially underlying product defect to which the patient referred. Further 3 cases $(27.3 \%)$ were not completely elucidated because in none of these cases the test strips and only in 2 cases the analyzers were sent back to the manufacturer for investi- gation of the complaint. In cases where the original test strips of the patients were not available for further investigation manufacturers tested retained test strips of the same batches which showed no product failure. However, these cases were defined to remain 'unclear' because it cannot be excluded that the patient's product might be affected by a product failure. Interestingly, in none of the cases related to OTC products for coagulation testing a user error has been identified as the underlying cause of the reported product failure.

Nine reports were related to reagents and test kits $(n=6)$ or control materials $(\mathrm{n}=3)$ for hematological diagnostics. In all cases product failures were confirmed by the investigations of the manufacturers (table 3). In detail, these were bacterial contaminations of the reagent (all cases affecting control materials), manufacturing errors (e.g. penetration of air into the vial followed by reagent oxidation, formation of particles which were not removed by a filtration step), material defects (insufficient stability of the used antibody, weak fluorescence of the labeled antibody, impaired stability of the fluorochrome bound to an antibody), and a packaging error (packing an incorrect instruction for use to the reagent). The typical appearance of product failures was very similar in all of these cases and characterized by an occurrence of falsely low or falsely high test results, an impaired reagent stability followed by erroneous results, an improper discrimination of cell populations consecutively followed by erroneous results, or a missing usability of the reagent.

Cases related to analyzers affected predominantly the analyzers themselves $(n=24)$ and only in 1 case a buffer solution re- 
Table 3. Product failures in 34 reported cases on IVD for hematological testing

\begin{tabular}{|c|c|c|c|c|}
\hline \multirow[t]{2}{*}{ Product failure } & \multicolumn{2}{|c|}{ Test $^{\mathrm{a}}$} & \multicolumn{2}{|c|}{ Analyzers ${ }^{b}$} \\
\hline & $\mathrm{n}$ & $\%$ & $\mathrm{n}$ & $\%$ \\
\hline Sum & 9 & 100.0 & 25 & 100.0 \\
\hline No product failure & 0 & 0.0 & 0 & 0.0 \\
\hline Unclear product failure & 0 & 0.0 & 0 & 0.0 \\
\hline User error & 0 & 0.0 & 2 & 8.0 \\
\hline Product failure & 9 & 100.0 & 23 & 92.0 \\
\hline Material defect & 3 & & 1 & \\
\hline Software bug & 0 & & 17 & \\
\hline Calibration error & 0 & & 0 & \\
\hline Mechanical error & 0 & & 0 & \\
\hline Lack of specification & 0 & & 0 & \\
\hline Manufacturing error & 2 & & 0 & \\
\hline Incorrect instructions for use & 0 & & 1 & \\
\hline Non-microbial contamination & 0 & & 0 & \\
\hline Packaging error & 1 & & 0 & \\
\hline Microbial contamination & 3 & & 0 & \\
\hline Interference by other substances & 0 & & 1 & \\
\hline Constructional fault & 0 & & 3 & \\
\hline Labeling error & 0 & & 0 & \\
\hline Root cause not identified & 0 & & 0 & \\
\hline
\end{tabular}

a Reagents, calibrators and controls.

${ }^{\mathrm{b}}$ Analyzers and consumables (cuvettes, solutions).

quired for the use of the analyzer. In 23 cases manufacturers were able to confirm a product failure, 2 further cases were caused by a user error (lack in the maintenance of the analyzer due to a missing cleaning of the barcode reader by the user although this was stated in the manufacturer's instruction for use) (table 3). The most frequent causes of analyzer failures were software bugs resulting in a risk for sample misidentification, missing values and zero values as well as erroneous results (e.g. due to erroneous calculation of dilution factors, calculation errors after manual data entry, calibration errors, failure of cell discrimination, errors in data acquisition, overestimation of cell populations, missing flags in case of analytical problems). Other causes were design-related product failures (malfunction of the analyzer's mix head under specific combinations of hard- and software; resonance of the syringe drive motor, discrepancy between the rubber stopper of specific blood tubes and the analyzer) resulting in erroneous results, an analytical interference (erroneous platelet results in one patient after chemotherapy), an insufficient instruction for use (warning for flags regarding blasts and platelets not described clearly enough), and a material defect (release of a chemical compound from the plastics of the container of a buffer solution required for the analyzer causing erroneous results).

\section{Corrective Actions}

In the group of tests, reagents, control materials and calibrators of professional-use IVD for coagulation testing, corrective actions were performed in 25 cases $(78.1 \%)$. No such measures were performed in 1 case, and in further 6 cases there were no measures in the German market because the affected products or batches were not distributed in Germany (table 4). The number of cases with corrective actions included 5 cases in which the underlying root cause was not identified. Corrective measures were above all customer information and recall; the latter took place even in combination with customer information. Another large group of corrective measures were modifications of the manufacturing process and the quality management system. These included - dependent on the underlying or assumed root cause - e.g. optimization of the control of raw materials used for the reagents, increase of in-process controls, implementation of more stringent criteria for product release, implementation of stress testing of the products prior to their release, implementation of visual controls in the manufacturing process, improvement of hygienic standards, qualification of the employees involved in product manufacturing, modification of the lyophilization process and the process of closing the vials, implementation of better standard operating procedures for reagent formulation, and optimization of the coating process of reactant-coated particles. Further corrective actions were modifications of the instructions for use (e.g. recommendations on product handling and stability, recommendations on specific combination of reagent batches, modification of the instruction for the test application on analyzers, limitations of the usage, implementation of additional washing steps), changes of the used raw materials (e.g. use of another glass type for the reagent vials, use of another thrombin and another factor Xa as raw materials, use of raw plasma isolated by another method for prevention of preactivation of the coagulation system), modifications of labeling (reduction of the product shelf life) as well as a software upgrade (regarding the data on the reagent chip).

Corrective actions were also performed in 9 cases $(90.0 \%)$ related to failures of analyzers and corresponding consumables. In 1 case the German market was not affected by the corrective action because the product was not distributed in Germany (table 4). Corrective actions were most frequently customer information and product recalls, the latter most commonly software upgrades due to underlying software bugs. In all cases software upgrades were preceded by customer information to immediately reduce the product-related risk. Other corrective measures were modifications of the manufacturing process and the quality management system (prevention of the chemical contamination of cuvettes, further validation of the melting process for cuvettes) as well as a modification of the instructions for use (modifications of the analyzer settings provided in the instructions for use).

In the group of systems for self-testing of coagulation corrective actions were taken in 6 of 11 cases, i.e. in all cases in which a product failure had been proven by the investigations of the manufacturers (table 4). On the other hand, no corrective actions were performed in cases where a product defect had been excluded or remained unclear. The corrective actions re- 
Table 4. Corrective actions in 53 cases of IVD for coagulation testing (multiple entries in case of corrective actions)

\begin{tabular}{|c|c|c|c|c|c|c|c|c|}
\hline & \multicolumn{2}{|c|}{$\begin{array}{l}\text { Professional-use } \\
\text { product test }\end{array}$} & \multicolumn{2}{|c|}{$\begin{array}{l}\text { Professional-use } \\
\text { product analyzer }\end{array}$} & \multicolumn{2}{|c|}{$\begin{array}{l}\text { All professional- } \\
\text { use products }\end{array}$} & \multicolumn{2}{|c|}{ OTC products } \\
\hline & $\mathrm{n}$ & $\%$ & $\mathrm{n}$ & $\%$ & $\mathrm{n}$ & $\%$ & $\mathrm{n}$ & $\%$ \\
\hline Number of cases & 32 & 100.0 & 10 & 100.0 & 42 & 100.0 & 11 & 100.0 \\
\hline No corrective actions $\mathrm{a}^{\mathrm{a}}$ & 7 & 21.9 & 1 & 10.0 & 8 & 19.0 & 5 & 45.5 \\
\hline Corrective actions & 25 & 78.1 & 9 & 90.0 & 34 & 81.0 & 6 & 55.5 \\
\hline Product recall / batch recall & 18 & & 6 & & 24 & & 5 & \\
\hline Stop of marketing & 0 & & 0 & & 0 & & 0 & \\
\hline Change of design & 0 & & 0 & & 0 & & 0 & \\
\hline $\begin{array}{l}\text { Modification of production and/or qual } \\
\text { management }\end{array}$ & 19 & & 2 & & 21 & & 4 & \\
\hline Customer information ${ }^{\mathrm{b}}$ & 25 & & 9 & & 34 & & 5 & \\
\hline Modification of the instruction for use & 5 & & 1 & & 6 & & 1 & \\
\hline Software-update & 1 & & 5 & & 6 & & 1 & \\
\hline Modification of labeling & 2 & & 0 & & 2 & & 0 & \\
\hline Modification of raw material & 4 & & 0 & & 4 & & 0 & \\
\hline Customer education $^{\mathrm{c}}$ & 0 & & 0 & & 0 & & 0 & \\
\hline
\end{tabular}

${ }^{a}$ Corrective actions which took place only outside the German market were considered as no corrective action.

${ }^{\mathrm{b}}$ Customer information with / without combination to recalls (in cases of product or batch recalls a customer information is obligatory.

${ }^{c}$ Education of a single customer e. g. after a user error was not defined to be a customer education. lated most frequently to test strip failures $(n=5)$ and included product recalls, customer information, modifications of production and/or quality management (additional and improved testing of bulk materials, improvement of the perforation process of the test strip packaging, increased number of inprocess controls, modification of product testing prior to release), and a modification of the instructions for use (information on the potential interference of lupus anticoagulant). In case of an analyzer failure due to an erroneous software algorithm, the corrective action included a product recall, a customer information and a software upgrade.

Corrective actions in tests and control materials of IVD for hematological diagnostics were performed in 8 (88.9\%) cases; in 1 more case there was no corrective action in Germany because the product was not distributed in the German market and our analysis was only made for the German market (table $5)$. The most frequent corrective actions were customer information and product recall. Modifications of manufacturing or quality management process included e g. the improvement of hygiene standards within the manufacturing process, education of employees involved in the manufacturing process, implementation of larger sample numbers and volumes for microbiological controls, implementation of an additional filter step as well as an increased stability testing and stress testing of the product. Further corrective actions were a distribution stop of the affected product (the manufacturer decided to replace the product by another one with an improved formulation), a modification of the product design, a modification of product labeling (reduction of the shelf life time) as well as a change of the used raw material (table 5).

In $23(92.0 \%)$ of the cases related to hematological analyzers and their consumables corrective actions were performed. As expected, these were different from those settled in the group of tests, reagents and control materials. The most frequent corrective actions were customer information and recall, the most common cause of the latter was a software upgrade which was preceded by a customer information for immediate risk reduction. Other corrective actions were modifications of the instructions for use, changes of design (modification of the hardware) and used materials (use of other materials in the analyzer, use of another plastic material for the container of the buffer solution), modification of labeling as well as stop of marketing. Only in the 2 cases caused by user errors no corrective measures were performed (table 5).

Corrective actions are typically performed in cases in which a product failure is confirmed by the investigations of the manufacturers. However, they may also be settled in cases where the underlying root cause of the product failure remains unclear, investigations reveal another product failure different from the reported one, or in case of user errors if the manufacturer decides to optimize the instructions for use even though the user error stands not in relation to missing information in the instruction for use. In our above mentioned analyses we did not differentiate with respect to these aspects. However, such an analysis may give some more information on the process of complaint management for ensuring product safety. In 6 cases of professional-use IVD for coagulation testing, the investigations of the manufacturers revealed no distinct root cause of the confirmed product failures. However, the manufacturer decided to perform corrective measures in all cases from which one took place outside the German market only. In contrast, in OTC products for coagulation testing 
Table 5. Corrective actions $n=34$ cases of IVD for hematological testing (multiple entries in case of corrective actions)

\begin{tabular}{|c|c|c|c|c|c|c|}
\hline & \multicolumn{2}{|c|}{ Test $^{\mathrm{a}}$} & \multicolumn{2}{|c|}{ Analyzers ${ }^{\mathrm{b}}$} & \multicolumn{2}{|c|}{ All products } \\
\hline & $\mathrm{n}$ & $\%$ & $\mathrm{n}$ & $\%$ & $\mathrm{n}$ & $\%$ \\
\hline Number of cases & 9 & 100.0 & 25 & 100.0 & 34 & 100.0 \\
\hline No corrective actions ${ }^{\mathrm{c}}$ & 1 & 11.1 & 2 & 8.0 & 3 & 8.8 \\
\hline Corrective actions & 8 & 88.9 & 23 & 92.0 & 31 & 91.2 \\
\hline Product recall / batch recall & 7 & & 20 & & 27 & \\
\hline Stop of marketing & 1 & & 1 & & 2 & \\
\hline Change of design & 1 & & 2 & & 3 & \\
\hline $\begin{array}{l}\text { Modification of production and/or } \\
\text { quality management }\end{array}$ & 5 & & 0 & & 5 & \\
\hline Customer information $^{\mathrm{d}}$ & 8 & & 22 & & 30 & \\
\hline Modification of the instruction for use & 0 & & 5 & & 5 & \\
\hline Software-update & 0 & & 18 & & 18 & \\
\hline Modification of labeling & 1 & & 1 & & 2 & \\
\hline Modification of raw material & 1 & & 2 & & 3 & \\
\hline Customer education $^{\mathrm{e}}$ & 0 & & 0 & & 0 & \\
\hline
\end{tabular}

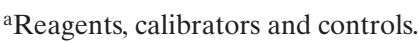

${ }^{\mathrm{b}}$ Analyzers and consumables (cuvettes, solutions).

${ }^{c}$ Corrective actions which took place only outside the German market were considered as no corrective action.

${ }^{\mathrm{d}}$ Customer information with / without combination to recalls (in cases of product or batch recalls a customer information is obligatory.

${ }^{e}$ Education of a single customer e. g. after a user error was not defined to be a customer education. no corrective measures were performed in all cases of unclear product failure. Finally, in the group of IVD for hematological diagnostics the 2 reports on product failure due to user errors were not followed by corrective actions.

\section{Discussion}

Until end of 2005 a total number of 653 cases was reported to the BfArM, and the annual number of reports is still increasing. In addition, a minor number of cases $(\mathrm{n}=138$; Halbauer J, 2007, personal communication) were reported between January 2002 and December 2005 to the PEI because these regarded to IVD for immune hematology, tissue typing and infective diseases listed in Annex II parts A and B of the Directive $98 / 79 /$ EC. The observed strong increases of reports to the BfArM after December 2001 and 2003 can be explained by the dates of the implementation of MPG in January 1, 2002 [2] and of MPSV in June 24, 2002 [3] as well as by the end of the transitional arrangements for labeling of IVD with the CE mark and marketing of unlabeled products as described in $\mathrm{Di}$ rective 98/79/EC and MPG [1, 2]. Another possible explanation for the continuous increase of reports is an increasing publicity and acceptance of the surveillance system. Based on the observation of increasing report numbers and the experience of the last years, the authors suggest that the European system for market surveillance is well functioning. However, it should be considered that there is an unknown number of unreported cases. Unfortunately, the relevance of this underreporting cannot be estimated because number and batch sizes of products in the German market are not known (in contrast to medicinal products medical products and IVD require no approval prior to marketing), and according to the German regulations it is not in the responsibility of the BfArM to make inspections of manufacturers and laboratories. In view of the BfArM there might be further optimization of the European surveillance system in some details. For example, the advertence especially of users for the legal regulations should be ameliorated, the establishment of the European database on medical products should be enforced, and uniform criteria and procedures for information of the other CAs and the public by vigilance reports and publication of field-corrective actions performed by the manufacturers (e.g. recalls and customer information due to product failures) e.g. on the homepages of the CAs should be established.

The majority of the reported cases $(n=491,75.2 \%)$ were products for professional use, including 42 and 34 cases of products for analysis of coagulation and hematological parameters, respectively. The latter represent a relevant proportion of all IVD for professional use $(n=76,15.5 \%)$. Additionally, 11 cases were reported relating IVD for self-testing of coagulation, reflecting $6.8 \%$ of all reported cases on OTC products ( $\mathrm{n}=162,24.8 \%$ of all cases reported for IVD) [8]. However, these products are also very important because treatment with oral anticoagulants bears a relevant risk for the patient in cases of over- and underdosage of the drug which causes bleeding and thromboses.

Our data demonstrate large differences between IVD for professional use and those for lay use in respect to the source of the report to the BfArM and the frequency of confirmed 
product failures. Reports related to professional-use IVD came predominantly from manufacturers or their distributors (the latter only to a minor number) and to a minor number from national or European CAs $(n=62,81.6 \%$ and $n=14$, $18.4 \%$, respectively). No reports were received from other sources, especially users. There was obviously no difference between professional-use IVD for coagulation testing and hematological diagnostics (reports from manufacturers vs. reports from CAs; products for coagulation testing: $\mathrm{n}=35 \mathrm{vs}$. $\mathrm{n}=7$; products for hematological analysis: $\mathrm{n}=27$ vs. $\mathrm{n}=7$; $\mathrm{p}=0.192$ ). In contrast to professional-use products for coagulation testing, reports related to corresponding OTC products came more frequently from users $(\mathrm{n}=3,27.3 \%)$; however this difference was not significant. The other sources of notifications in the latter group were manufacturers or their distributors and CAs $(n=7,63.4 \%$ and $n=1,9.1 \%$ respectively). The observed differences regarding the source of notification confirm the results of prior analyses on IVD for professional use and lay use and can be explained by different procedures in reporting product failures [7-10]. Professional users usually report their complaint to the manufacturer or the distributor who performs some investigations regarding the complaint and - if it can be confirmed and is considered to be relevant for reporting according the criteria for reporting of incidents $[3,5-7]$ - reports the product failure to the BfArM. In contrast, lay users report directly to the BfArM or more frequently contact their local pharmacy in cases of product failures. The latter reports it to the BfArM or forward the complaint to the Drug Commission of the German Pharmaceutical Association which transmits it to the BfarM [8].

Even though the number of cases related to OTC products for coagulation testing is small $(\mathrm{n}=11)$, we found also some differences regarding the frequency of product failures in comparison to professional-use products. The relative proportion of proven product failures confirmed by the manufacturers is higher in professional-use products for coagulation testing than in OTC products ( 97.6 vs. $54.5 \% ; \mathrm{p}=0.001$ ); no difference was found between professional-use products for hematological testing and for coagulation testing (97.6 vs. $94.1 \%$; $\mathrm{p}=0.608)$. These data stand in ample agreement to data published for OTC products for self-monitoring of blood glucose, reporting a low number of proven not user-related product failures $(27.3 \%)$ and a high number of cases in which the existence of a product failure remained unclear $(25.8 \%)$ or was caused by a user error (22.7\%) [8]. Interestingly, no user errors were reported for OTC products for coagulation testing. However, the lack of user errors in this group might be caused by the low number of cases in our study. The difference regarding the numbers of proven product failures between professional-use IVD and OTC products can be easily explained by the user groups and the way of the notification to the BfArM. Even though patients who are self-monitoring their coagulation are well trained, it can be assumed that professional users have a much better education for the use of the
IVD. In principle, this reduces the number of user errors and unclear cases in the group of professional-use IVD. Regarding the cases remaining unclear, one should consider that in the group of OTC products cases remained unclear because the affected products were not or not completely (analyzer and test strips) available for investigation by the manufacturers, whereas in the group of professional-use products cases remained unclear because even intensive investigations of the manufacturers were not able to elucidate the underlying root cause which might be a consequence of the complexity of the reagent formulations and the possible interactions of their components. In addition, as described before lay users typically report directly or via their pharmacies to the BfArM, whereas professional users contact the manufacturer or the distributor which serves as a filter while evaluating the complaint for its relevance.

Another point of interest is the distribution of failures related to test strips to failures related to analyzers. Even though the number of proven product failures in OTC products for coagulation testing $(n=6)$ in the observation period remained low, test strips appear to bear a higher risk for product failures than analyzers ( 5 vs. 1 case), confirming the data previously published for OTC products for monitoring of blood glucose [8]. Further differences of frequency and type of the reported failures were found between products for testing of coagulation parameters and those for testing of hematological parameters. Professional-use tests, reagents, calibrators and control materials for coagulation testing were much more frequently affected than the corresponding analyzers (32 (76.2\%) vs. 10 $(23.8 \%)$ cases). In contrast, hematological analyzers are more prone to product failure than hematological tests, reagents, calibrators and control materials $(25$ (73.5\%) vs. 9 (26.5\%) cases). This reflects the large differences between both product groups (hematological products vs. coagulation products $\mathrm{p}$ $\leq 0.001)$. Potential explanations for this observation are the differences in the chemistry of the reaction in case of the tests and the required properties of the analyzer software in case of the analyzers. Assays for blood coagulation monitoring are commonly based on kinetic measurement of reactions of proteolytic enzymes and inhibitory proteins in the coagulation cascade. Any variation of the reagent composition due to a formulation or production error or use of an inadequate (e.g. unstable or pre-activated) raw material will be followed by an unstable or incorrect reaction and consecutively by erroneous results. Due to the complexity of the coagulation system, functional (i.e. kinetic) tests for measurement of coagulation parameters will be influenced to a higher extent than other tests based for example on immunological means for measurement of an analyte. Even though the reaction conditions for coagulation assays are very complex, analyzers for its measurement are relatively simple when compared to other, for example hematological analyzers. In principle, this hypothesis is confirmed by the observation of the high rate of analyzer failures due to software bugs in the group of IVD for hematological 
diagnostics and the low frequency of product failures related to tests and control materials in this group. It is likely that the complex software algorithms which serve for identification, differentiation and even quantification of blood cells are more prone to errors than software algorithms for determination of other parameters, for example the coagulation reaction.

In most of the cases reported to the BfArM, corrective actions were performed by the manufacturers. In principle, there are two types of corrective actions. The first one has the goal to reduce the risk of medical products or IVD which are already on the market and are or even may be affected by the reported product failure. This group of corrective actions includes recalls, customer information and distribution stop of the affected product. Another type of a corrective action is the preventive action by which the manufacturer tries to reduce the risk of products which will be delivered into the market in the future. The latter type of a corrective action includes for example changes of the affected raw materials, modifications of the product design as well as modifications of the manufacturing process or the quality management system. However, there is often no discrimination between both types of action which are often summarized as 'corrective action / preventive action' (CAPA). Therefore, we did also not differentiate in our analyses between both types of measures. However, the obtained data clearly demonstrate that tests and reagents are mainly affected by material defects and manufacturing errors, whereas analyzer failures are typically caused by software bugs. In consequence, the corrective actions in reagent-related failures focus predominantly on changes of the used raw materials and modifications of the manufacturing process or the quality management system, whereas software upgrades were the typical corrective measures in analyzer failures. However, no detailed data about the efficiency of the corrective actions taken by the manufacturers are available because in Germany the surveillance of the corrective actions is not in the responsibility of the Federal State authorities but in the responsibility of the local authorities of the German countries. Even though detailed information is lacking, the experience of the BfArM shows that the corrective actions seem to be effective because there is a very little number of repeated complaints due to the same product failure.
In summary, our data describe the experience of the BfArM in the European market surveillance system on IVD for the first 6 years. It is likely that our data are compromised by an underreporting of issues related to IVD from users and/or manufacturers and their distributors which cannot be estimated in its extent. In addition, several points of the surveillance system should be subject to further optimization (e.g. information exchange between CAs, publishing of corrective actions of manufacturers). However, our data indicate that the European surveillance system for medical products (including IVD) is an established tool for ensuring the safety of these products. Furthermore, our data suggest that there are large differences between the various groups of IVD, e.g. professional-use products vs. OTC products for coagulation testing, professional-use IVD for coagulation testing vs. IVD for hematological diagnostics as well as test strips vs. analyzers for coagulation testing in OTC products. The results of our analysis demonstrate that reagents (including calibrators and controls) for coagulation testing bear a higher risk for users than the corresponding analyzers, whereas in case of IVD for hematological diagnostics analyzers are more at risk than reagents which might be caused by specific problems of reagents and analyzers. In both groups the risk to users typically affected the patients because incorrect test results may delay or prevent diagnostics of diseases or may cause an incorrect therapy of patients, whereas personnel performing the laboratory analyzes was not at risk (e.g. by electrical shock or infection). Issues related to the reagents were commonly caused by material defects and production errors affecting reagent stability and results, whereas analyzer failures were typically caused by software bugs resulting in erroneous results due to errors in measurement and calculation of the results as well as sample misidentification. Additionally, in OTC products for coagulation testing test strips were more frequently subject of product failures than analyzers, confirming our data obtained in OTC products for monitoring of blood glucose [8]. In summary, our data demonstrate the weak points of IVD in the different product groups which may help manufacturers and users to further improve product safety.

\section{References}

1 Directive 98/79/EC of the European Parliament and of the Council of 27 October 1998 on in vitro Diagnostic Medical Devices. Official Journal L 331, p. 1-37. http://europa.eu.int/eur-lex/en/index.html.

2 Medizinproduktegesetz (MPG) in der Fassung der Bekanntmachung vom 7. August 2002. BGBl. I;2002:3146. www.bfarm.de/de/Medizinprodukte/ mp_recht/index.php.

3 Medizinprodukte-Sicherheitsplanverordnung (MPSV) vom 24. Juni 2002. BGBl. I 2002:2131. www.bfarm.de/de/Medizinprodukte/mp_recht/inde x.php.
4 Spitzenberger F, Edelhäuser R: Accreditation of medical laboratories in Europe: statutory framework, current situation and perspectives. Transfus Med Hemother 2006;33:384-392.

5 Guidelines on a Medical Devices Vigilance System. MEDDEV 2.12-1 rev. 4. http://europa.eu.int/comm/ enterprise/medical_devices/guidelinesmed/baseguide lines.htm.

6 Medical Devices Post Market Surveillance: National Competent Authority Report Exchange Criteria. GHTF/SG2/N20R10:2002. www.ghtf.org.

7 Die Anforderungen der Medizinprodukte-Sicherheitsplanverordnung für In-vitro-Diagnostika im Rahmen des Medizinproduktegesetzes. MedizinProdukteRecht 20024:120-133. www.vdgh.de.
8 Siekmeier R, Lütz J: Wie sicher sind Blutzuckermesssysteme? Erfahrungen des Bundesinstituts für Arzneimittel und Medizinprodukte mit den dort bis Ende 2005 eingegangenen Meldungen. Bundesgesundheitsbl Gesundheitsforsch Gesundheitssch 2006;49:1212-1218.

9 Siekmeier R, Will HG: German experience with the European vigilance system for in vitro diagnostic medical devices 1999-2002. Clin Chem Lab Med 2003;41:A87(abstract).

10 Siekmeier R, Will HG: Safety of lay use products German experience with the European vigilance system for in vitro diagnostic medical devices. Clin Chem Lab Med 2004;42:A151 (abstract). 